\title{
Designing environmentally friendly chemical processes with fugitive and open emissions
}

\author{
R.L. Smith ${ }^{\mathrm{a}, *}$, T.M. Mata ${ }^{\mathrm{b}}$, D.M. Young ${ }^{\mathrm{a}}$, H. Cabezas ${ }^{\mathrm{a}}$, C.A.V. Costa ${ }^{\mathrm{b}}$ \\ ${ }^{a}$ U.S. Environmental Protection Agency, Office of Research and Development, National Risk Management Research Laboratory, 26 West \\ Martin Luther King Drive, Cincinnati, OH 45268 USA \\ ${ }^{\mathrm{b}}$ Laboratory of Processes, Environment and Energy Engineering, Faculty of Engineering, University of Porto, Rua Dr. Roberto Frias, 4200-
} 465, Porto, Portugal

Received 30 July 2002; accepted 5 December 2002

\begin{abstract}
Fugitive or open emissions can dominate the potential environmental impacts of a chemical process. In this work the design and simulation calculations of a process provide an opportunity to visualize relationships between economic potentials and potential environmental impacts. The analysis of the economic and environmental effects of process alternatives are completed quickly and easily using order-of-magnitude costing techniques and the Waste Reduction algorithm for environmental evaluation. In the example studied, the hydrodealkylation of toluene, both the economic and environmental results point towards the alternative of recycling diphenyl to extinction, which is a form of pollution prevention by source reduction. As open emissions are eliminated, the importance of fugitive emissions is shown to increase. Finally, results show where economic optimum and minimal environmental impact designs occur, and therefore one can see tradeoffs between these designs.
\end{abstract}

Published by Elsevier Ltd.

Keywords: Fugitive; Leaks; WAR; Waste reduction algorithm

\section{Introduction}

Designing a chemical process normally includes aspects of economic and environmental disciplines. While the desire for profit (or minimal cost) leads to studying the economics, the desire to be safer and environmentally friendlier leads to examining the environmental releases from a process. Releases can be detrimental to the environment and at the same time have a negative effect on the economics. Studying these negative effects will increase our understanding of chemical processes. It is the knowledge produced from including both environmental and economic analyses that this work explores.

The more specific focus of this work is on methods to quickly and easily evaluate the economics and potential environmental impacts of a process that has both open and fugitive emissions. By making the methods quick

\footnotetext{
* Corresponding author. Fax: +1-513-569-7111.

E-mail address: smith.raymond@epa.gov (R.L. Smith).
}

and easy an engineer can use them at various stages of the development process. In particular, when one can examine processes early in their development, it is much easier to make substantial changes - changes that have large effects on both economic and environmental results. To describe the analyses methods and study alternative designs, we have chosen the hydrodealkylation of toluene as an example.

\section{Background}

There are a number of methods applied to the development of chemical process designs. To study alternatives at an early stage one can use heuristic methods [1], which employ a hierarchical procedure for developing flowsheets. For waste minimization a hierarchical approach has been suggested by Rossiter and Klee [2]. These hierarchical methods can be contrasted with mathematical programming techniques such as mixed-integer nonlinear programming, which has been combined with a hierarchical technique to develop conceptual designs 
[3]. Once a conceptual design is developed, one can focus on more details by performing simulations (e.g. with ASPEN PLUS, CHEMCAD, HYSYS, or PRO/II for example). These simulations can provide a more rigorous and detailed analysis that increases confidence in a design.

The engineering calculations that specify conceptual designs lead to economic analyses of a process. These analyses can be done at different levels. One can do an order-of-magnitude estimate or add finer details to get a more accurate evaluation. In this work an order-ofmagnitude estimate will be done, as has been discussed extensively for the hydrodealkylation of toluene [1].

To evaluate the potential environmental impacts of a process, the work of Young and Cabezas [4] and Young et al. [5] on the Waste Reduction (WAR) algorithm is extended to include both open and fugitive emissions. (A paper by Cano-Ruiz and McRae [6] reviews environmentally conscious design.) The WAR algorithm uses a database of potential environmental impacts for $1600+$ chemicals to evaluate streams that cross the system boundaries. The mass flowrates of these streams are multiplied by database values that reflect chemical potency to obtain potential environmental impacts (PEI). The database values include local impact categories such as human toxicity by dermal/inhalation and ingestion routes, terrestrial toxicity and aquatic toxicity. Regional impact categories include photo-chemical oxidation and acidification, while global categories include ozone depletion and global warming. Each of these categories has scores that have been normalized within the category, while weighting factors are applied between categories. Note that in this work all of the weighting factors are set to 1.0 (equal to each other), and we assume that the impacts from the various categories are additive. These assumptions could be altered by a decision-maker. Besides these assumptions, it is important to consider where emissions originate. Among the various exit streams from a process, certain ones are known sources of waste, while other sources come from leaks through valves, flanges, etc. This work studies the tradeoffs between economics and environmental impacts, and also the importance of fugitive versus open emissions.

\section{Designing the hydrodealkylation process}

Open emissions, those releases that are purposely allowed to occur, continue to decline in importance in the chemical process industries because of regulations and voluntary practices. With this decline in open emissions we can expect that fugitive emissions are gaining in importance. However, it is unclear what fraction of the emissions, and more importantly the potential environmental impacts, are due to open or fugitive releases. Therefore, we will examine the amounts of open and fugitive emissions, assuming that $0.1 \%$ of each stream in a flowsheet is lost as a fugitive emission. This assumption can be refined at a later time (e.g. [7]).

The process under consideration here is the hydrodealkylation of toluene to form benzene. This process often follows a reformer in a refinery, where the reformer turns cyclic compounds into benzene, toluene, and xylene (among many other species). When these aromatics are separated from the reformate they can be purified and sold or used as individual chemicals. The demand for such chemicals can have a specific (but changeable) distribution, and the hydrodealkylation of toluene is one process for altering the supply distribution. This process, shown in Fig. 1, uses toluene and hydrogen feeds to form benzene. The resulting product is benzene and an undesired byproduct diphenyl, which is formed in series from benzene. An alternative design to that shown in Fig. 1 is to recycle diphenyl with toluene back to the reactor system. There the diphenyl builds up to an equilibrium level according to a reversible reaction with benzene. Descriptions of these processes, including a selectivity relationship used in spreadsheet calculations, are given by Douglas [1], while the kinetics for the simulations are from Luyben [8].

Analyses of the economics for the hydrodealkylation processes are shown in Fig. 2a, where successive levels of detail are added to the process flowsheet structure. Included in the economic potential (EP) are the annualised profits minus costs of chemicals and equipment, including hazardous waste treatment (U.S. Army Corps of Engineers [9]) and fugitive emission costs. From Fig.

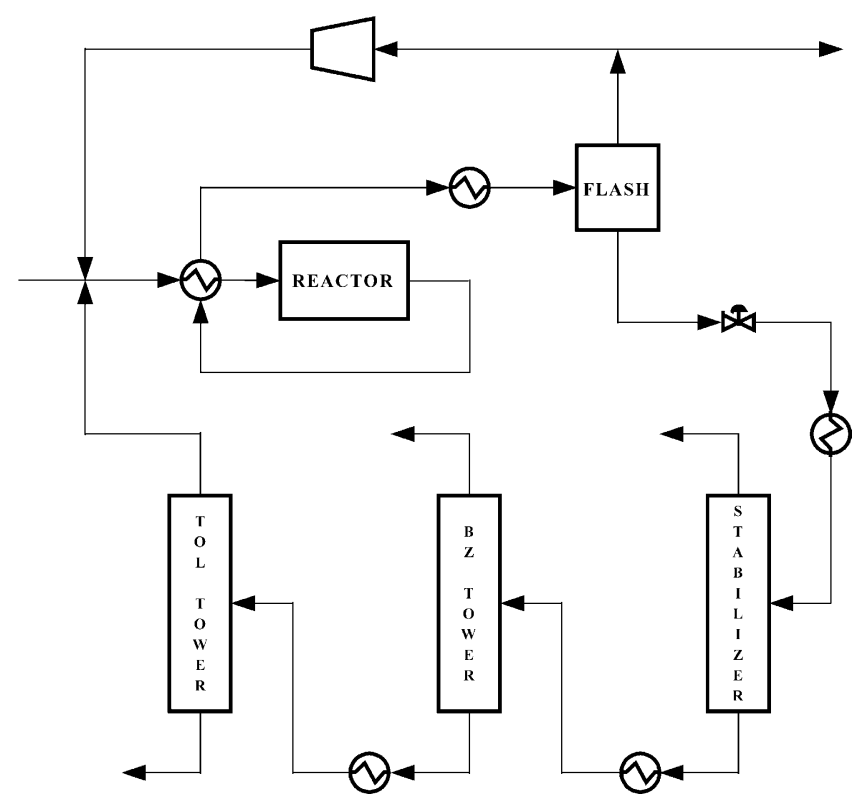

Fig. 1. Process flowsheet for the hydrodealkylation of toluene, with removal of diphenyl as the bottoms from the toluene tower. Toluene and hydrogen are fresh feeds to the process, while benzene is obtained as the tops from the benzene tower and hydrogen and methane leave the process through the purge and stabilizer tops streams. 

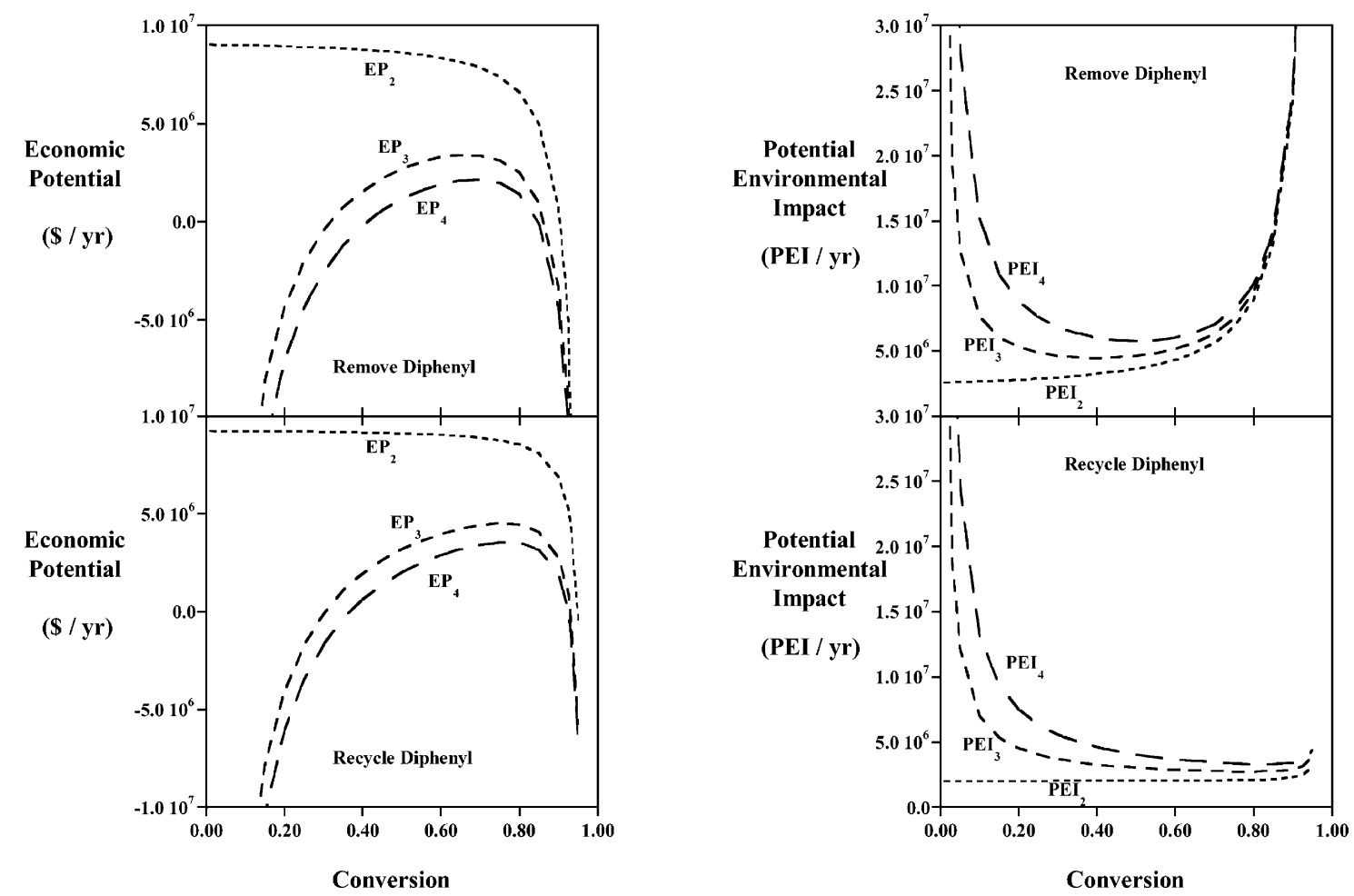

Fig. 2. (a) Economic potentials for the Input-Output $\left(\mathrm{EP}_{2}\right)$, Reactor-Recycle $\left(\mathrm{EP}_{3}\right)$, and Separation $\left(\mathrm{EP}_{4}\right)$ phases of a hierarchical design. (b) Potential environmental impacts for the same phases.

2a one can see that higher economic potentials can be obtained when diphenyl is recycled to extinction, and the optimum conversion increases to approximately 0.75 in this case as well.

Studying the potential environmental impacts (PEI) in Fig. 2b shows how increased levels of detail require more equipment and streams that increase the amount of fugitive emissions. The results indicate that recycling diphenyl reduces PEI considerably, especially at higher conversions. This result is due to the emission of diphenyl in the case where it is removed from the process. Even if the diphenyl is treated, it is considered to be emitted to the environment, as the best estimation of the effects of the treated emissions (without knowing the results of treatment) is to use the diphenyl PEI values themselves.

By considering the fugitive emissions from streams in the flowsheet one can focus attention on the most deserving areas. For instance, Fig. 3a shows five streams from the flowsheet according to the EP lost and PEI caused by their fugitive emissions. For both aspects it is most important to concentrate on the fugitive emissions of the recycle streams, followed by the reactor effluent, and other streams. While these streams follow a fairly regular pattern in terms of the importance of EP and PEI, it is quite possible that a valuable fugitive emission would have a small PEI or vice versa. Note that when the flowsheet is altered to consider the recycle of diphenyl to extinction that the toluene tower is not present, and the fugitive emissions from that source are eliminated.

One way to emphasize the differences in the results between the alternative designs is to present them as shown in Fig. 3b. Here, the EP lost due to environmental costs (hazardous waste treatment and fugitive emission losses) is graphed as a fraction of the value of the product. Thus, one spends as little as $1 \%$ of the product value on environmental aspects when diphenyl is recycled to extinction. However, when diphenyl is removed the costs are minimal at about $4 \%$, and this percentage increases as one moves towards the economic optimum conversion shown in Fig. 2a. Thus, preventing the pollution through source reduction is the best alternative, in terms of both the economics and the environment.

The amount of PEI that fugitive emissions represent is displayed in Fig. 3b, where the case of recycling diphenyl shows a larger fraction of PEI arising from fugitive emissions. Logically, when diphenyl is emitted openly, the fraction of PEI from fugitive emissions is lower. Thus, a figure such as this shows where one should focus efforts on reducing environmental impacts. As open emissions are reduced, more impact comes from fugitive emissions. 

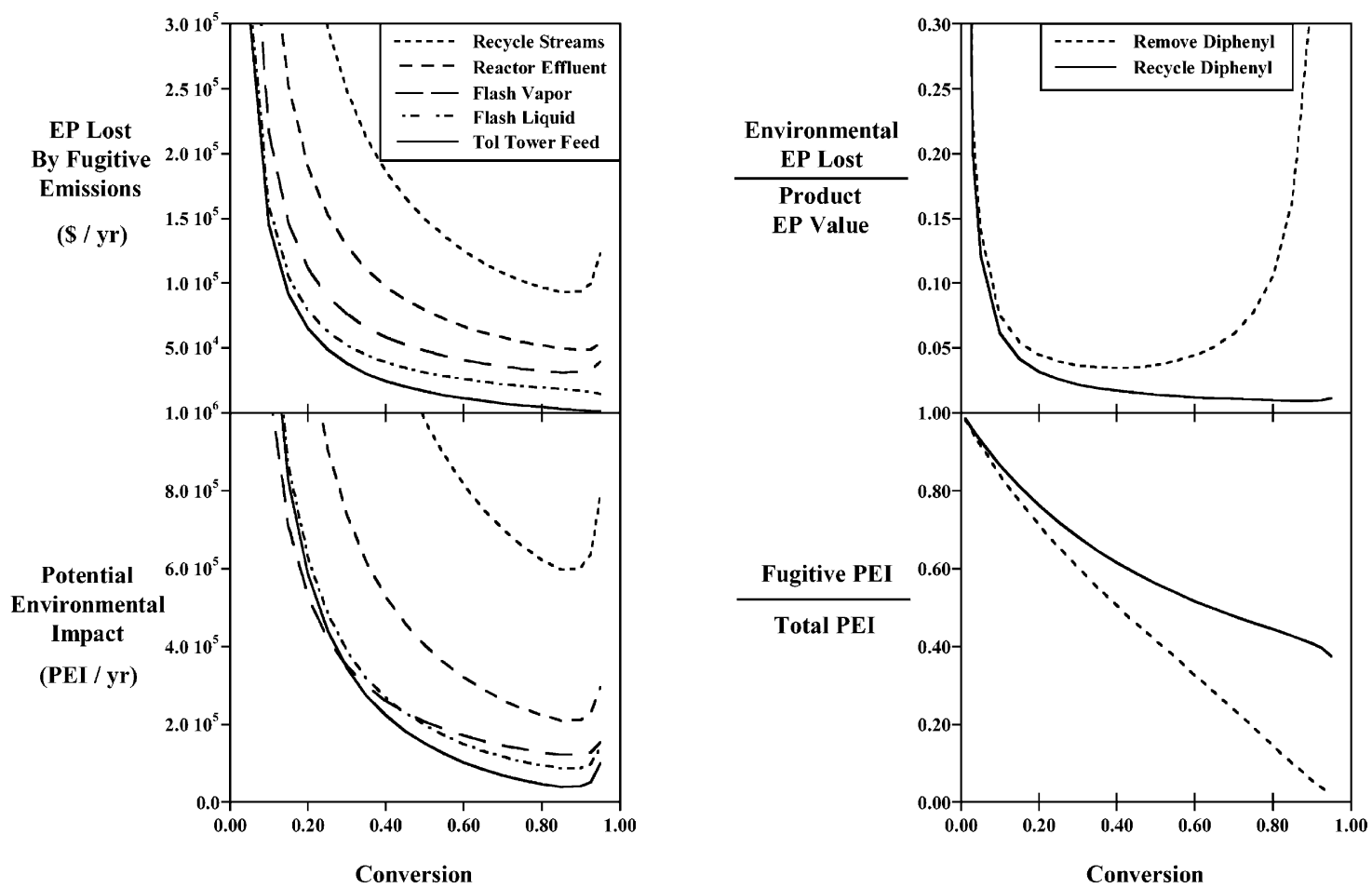

Fig. 3. (a) Economic potential lost and potential environmental impact of various streams for the case of removing diphenyl. (b) Hazardous waste treatment and fugitive emission costs per product value, and the fraction of potential environmental impact due to fugitive emissions.

\section{Simulating the hydrodealkylation process}

Once a process has been designed (in this case using heuristics and short-cut calculations such as perfect splits in towers), it is reasonable to refine the calculations through simulation. This was done for the hydrodealkylation process for the case where diphenyl was recycled to extinction, since it was found to be the better alternative in terms of both economic potential and potential environmental impact. These calculations assumed that a particular feed of toluene and hydrogen (mixed with methane) was available, which is a reasonable assumption following the reformer process. Equipment was maintained at specific sizes, except for the reactor system which was allowed to vary in size to alter the amount of conversion.

Results of the simulations define the needed heat exchangers and duties, as well as the recycle compressor, stream temperatures and pressures, and the flash and tower splits that can be obtained. Using these calculations the potential environmental impact for the recycle of diphenyl case shows a higher level (approximately $1.6 \times 10^{7} \mathrm{PEI} /$ year at 0.45 conversion) than that from the design calculations (See Fig. 2b). However, similar to the design calculations, the PEI decreases slightly with higher conversions, so the overall trend in the results is similar. One can expect a difference between such calculations because the simulator uses more complete models (and better thermodynamics and kinetics than the assumptions used for the design calculations), and the simulator uses a larger number of streams, which adds to the fugitive emissions based on our assumption of $0.1 \%$ of each stream.

The rigorous calculations done with a simulator add to our confidence in the conceptual designs. After performing these calculations we can complete a sensitivity analysis on parameters, design specific experiments, or move forward to scale up a process depending on our engineering judgment. The economic and environmental analyses could also be refined. In particular, the potential environmental impacts can be considered according to each category of impact as well as the specific location for the process. These possible next steps build on the design calculations and simulations performed in this work.

\section{Conclusions}

A design and simulation of a process for the hydrodealkylation of toluene to produce benzene provides an example showing economic and environmental relationships. For this process both the economic and environmental results point towards the alternative of recycling diphenyl to extinction. While even lower potential environmental impacts are obtained by increasing the conversion to higher levels, the economic optimum occurs around 0.75 conversion. Results indicate which streams have potentially the greatest losses in terms of economic potential and potential environmental impact 
due to fugitive emissions, and therefore one knows which streams to focus on for fugitive emissions. A simple display of results as fraction of product value spent on environmental costs and fraction of potential environmental impact from fugitive emissions shows where emphasis should be placed. In particular, as open emissions are eliminated fugitive emissions become more and more important.

\section{References}

[1] Douglas JM. Conceptual design of chemical processes. New York: McGraw-Hill, 1988.

[2] Rossiter AP, Klee H. Hierarchical process review for waste minimization. In: Rossiter AP, editor. Waste minimization through process design. New York: McGraw-Hill; 1995.
[3] Daichendt MM, Grossmann IE. Integration of hierarchical decomposition and mathematical programming for the synthesis of process flowsheets. Comput. Chem. Eng 1997;22(1-2):147-75.

[4] Young DM, Cabezas H. Designing sustainable processes with simulation: The Waste Reduction (WAR) Algorithm. Comput. Chem. Eng 1999;23:1477-91.

[5] Young DM, Scharp R, Cabezas H. The Waste Reduction (WAR) Algorithm: environmental impacts, energy consumption, and engineering economics. Waste Management 2000;20:605-15.

[6] Cano-Ruiz JA, McRae GJ. Environmentally conscious chemical process design. Annu. Rev. Energy Environ 1998;23:499-536.

[7] EPA 453/R-93-026. Protocol for equipment leak emission estimates. Research Triangle Park, NC: Office of Air Quality Planning and Standards, 1993.

[8] Luyben WL. Effect of kinetic, design, and operating parameters on reactor gain. Ind. Eng. Chem. Res 2000;39:2384-91.

[9] U.S. Army Corps of Engineers. Report on treatment, storage, and disposal facilities for hazardous, toxic, and radioactive waste. Omaha, NE: HTRW Center of Expertise, 1998. 